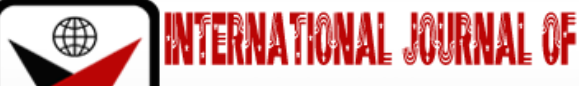

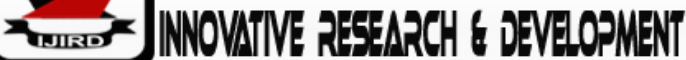

ISSN 2278 - 0211 (Online)

\section{Existing Building Evaluation against SNI (Indonesian National Standards) 1726-2019 Concerning Earthquake Resistance Planning Procedures for Building and Non-Building Structures}

\section{Tranggono}

Lecturer, Department of Civil Engineering, Yos Soedarso University,Surabaya, Indonesia

\begin{abstract}
:
With the issuance of the 2019 SNI 1726-2019 Earthquake Regulations, it is necessary to recalculate the strength of the existing buildings designed with SNI 1726-2012. The building that becomes the case study is a hospital located in Mojokerto City, East Java, Indonesia. This study aims to determine the strength of the existing building against the provisions in SNI 1726-2019. The method of calculation is by evaluating the workload of the foundation, calculating the amount of deviation between levels and the effect of $P$ - $\Delta$ that occurs. The building was built in 2018 and designed using the SNI 1726-2012Earthquake Regulations which are based on the 2017 Earthquake Map. The results of the recalculation are: 1 . The deviation between levels in the X-direction on the $1^{\text {st }}$ floor and $2^{\text {nd }}$ floor is greater than the allowable deviation, while on the $3^{\text {rd }}$ and $4^{\text {th }}$ floor it is smaller than allowed. The Y-direction deviation on all floors is smaller than allowable; 2 Workload on the foundation at all points of the column with a combination of gravity load and earthquake load is smaller than the allowable bearing capacity.
\end{abstract}

Keywords: SNI 1726-2019 Earthquake Regulations; Allowable bearing capacity for the foundation; Deviation between levels; Effect of $P-\Delta$.

\section{Introduction}

\subsection{Background}

The country of Indonesia, which is an archipelago, is one of the areas that has a very high level of risk of earthquake disasters because of its geographic location between four active tectonic systems, namely the Eurasian plate, the Indo-Australian plate, the Philippine plate and the Pacific plate, which are known to be as the most active plate. In the territory of Indonesia, the incidence of strong earthquakes that damage the average is 3 times in 2 years (Siddiq, 2014).

In every earthquake, there are often many victims of life and property. However, the large number of casualties during an earthquake is often caused by the collapse of the structure of the occupied buildings, whether residential buildings, offices or other types of buildings due to not being able to withstand the dynamic loads of the earthquake. Therefore, it is important to pay attention to the planning of earthquake-resistant buildings in Indonesia in order to reduce the risk of casualties in the event of an earthquake. Suwandojo Siddiq (Siddiq, 2014) suggests that efforts can be made to prevent damage to buildings due to earthquake disasters as follows: 1) planning new earthquake-resistant buildings; 2) Performing repairing, strengthening and retrofitting of existing buildings which are considered 'weak' so that they are strong against the impending earthquake forces; 3 ) repairing and strengthening damaged buildings (mild to moderate) due to the previous earthquake, so that their strength and rigidity can be returned, even stronger or more rigid.

Many factors cause and level of damage to building structures caused by the earthquake, including the configuration of the structural system, uneven distribution of loads and the lack of rigidity of the building structure (vertical and horizontal). Currently, the limitation of land is the main reason for the implementation of multi-story building planning. Such vertical building planning is, in fact, prone to earthquakes (Istiono and Khoe, 2020). Meanwhile, the potential risk of an earthquake in Indonesia is high. The regulations for the design of earthquake resistant building structures in Indonesia that are used refer to SNI 1726-2012. However, due to the large number of earthquakes with a large enough magnitude in Indonesia and causing damage to building structures and casualties, SNI 1726-2012 is deemed inappropriate to be applied as a guideline for earthquake-resistant building structure planning. Therefore it is necessary to renew so that SNI 03 1726-2019 is compiled as a regulation for planning new earthquake resistant building structures (Afnan et al, 2020).

The enactment of the SNI 1726-2019 Earthquake Regulations in 2019 causes changes in earthquake parameters which result in changes in the magnitude of the seismic force. This is what motivates the need to re-evaluate buildings that have been designed with the previous SNI 1726-2012 Earthquake Regulations with the latest SNI, namely SNI 1726-2019. 


\subsection{The Aim of the Study}

Make an evaluation of the strength of existing buildings designed with SNI 1726-2012 against the provisions for earthquake resistant buildings in SNI 1726-2019.

\subsection{Limitation of Research Problem}

The location of the building is in Mojokerto City, East Java Province, Indonesia. The building function is for a hospital with a building area of $15 \times 25 \mathrm{~m}^{2}$ and the number of floors is 4 floors. Soil conditions are soft. Main column dimensions $60 \times 60 \mathrm{~cm}^{2}$, transverse beam $40 / 70 \mathrm{~cm}^{2}$ and $30 / 60 \mathrm{~cm}^{2}$, spun pile foundation $\Phi 40 \mathrm{~cm}$ with a depth of $25 \mathrm{~m}$. The height of the 1st floor is $5 \mathrm{~m}$, while the floors above are typically $4 \mathrm{~m}$.

Evaluation is done by calculating: 1 . Foundation workload; 2. Deviation between levels; 3 . Effect of P - $\Delta$

\section{Research Method}

\subsection{The Stages of Analysis Carried Out in This Study Are as Follows:}

- Determine the building Risk Category

- Determining Priority Factor

- Determine the Earthquake Spectra Response Parameters for a short period $\left(\mathrm{S}_{\mathrm{s}}\right)$

- Determine the Earthquake Spectra Response Parameters for a period of 1 second $\left(\mathrm{S}_{1}\right)$

- Determining the Classification of Land Sites (SA - SF)

- Determining the Land Sites Coefficient Factor $\left(\mathrm{F}_{\mathrm{a}}, \mathrm{F}_{\mathrm{V}}\right)$

- Calculate the Design SpectralAcceleration Parameterfor a short period $\left(\mathrm{S}_{\mathrm{DS}}\right)$

- Calculate the Design SpectralAcceleration Parameterfor a period of 1 second( $\left.\mathrm{S}_{\mathrm{D} 1}\right)$

- Select the System danStructure Parameters $\left(\mathrm{R}_{1} \mathrm{C}_{\mathrm{d}}, \Omega_{0}\right)$

- Evaluation of structures system related toconfiguration irregularities

- Determine flexibility of diaphragm (flexible, semi-rigid, rigid)

- Determine the redundancy factor $(\rho)$

- Define the lateral force analysis procedure

- Calculating the lateral load

\subsection{Equivalent Static Load Analysis}

- Basic SeismikLoad $\rightarrow \mathrm{V}=\mathrm{C}_{\mathrm{s}} \mathrm{W}$

- EquivalentStatic Earthquake Force Distribution:

$\mathrm{F}_{\mathrm{X}}$

$\mathrm{C}_{\text {vx }}$

$\mathrm{F}$

$\mathrm{C}_{\mathrm{V}}$

$\mathrm{h}_{\mathrm{i}}$

$\mathrm{W}_{\mathrm{i}}$

$\mathrm{K}$ forequivalent static

\subsection{Analysis of Spectra Response}

- $\quad$ Determining Time of Vibration (T)

$\mathrm{T}_{\mathrm{S}}=\mathrm{S}_{\mathrm{D} 1} / \mathrm{S}_{\mathrm{DS}}$

$\mathrm{T}_{\mathrm{o}}=0,2\left(\mathrm{~S}_{\mathrm{D} 1} / \mathrm{S}_{\mathrm{DS}}\right)$

- $\quad$ Determining the Design Acceleration Spectrum Response $\left(\mathrm{S}_{\mathrm{a}}\right)$

For $\mathrm{T}<\mathrm{T}_{\mathrm{o}} \rightarrow \mathrm{Sa}=\mathrm{S}_{\mathrm{DS}} \mathrm{x}\left[0,4+0,6 \frac{\mathrm{T}}{\mathrm{To}}\right]$

For $\mathrm{T}<\mathrm{T}_{\mathrm{o}}<\mathrm{T}_{\mathrm{S}} \rightarrow \mathrm{Sa}=\mathrm{S}_{\mathrm{DS}}$

For $\mathrm{T}>\mathrm{T}_{\mathrm{S}} \rightarrow \mathrm{Sa}=\frac{\mathrm{SD} 1}{\mathrm{~T}}$

\subsection{Combination of Loading}

Combination ofUltimite Load

- $1,4 \mathrm{DL}$

- $1,2 \mathrm{DL}+1,6 \mathrm{LL}+0,5(\mathrm{~A}$ or $\mathrm{R})$

- $1,2 \mathrm{DL}+1,0 \mathrm{LL} \pm 1,6 \mathrm{~W}+0,5(\mathrm{~A}$ or $\mathrm{R})$

- $1,2 \mathrm{DL}+1,0 \mathrm{LL} \pm 1,0 \mathrm{E}$

- $0,9 \mathrm{DL} \pm 1,6 \mathrm{~W}$

- $0,9 \mathrm{DL} \pm 1,0 \mathrm{E}$

Combination of Service Load

- $1,0 \mathrm{DL}$

- $1,0 \mathrm{DL}+1,0 \mathrm{LL}$

- $1,0 \mathrm{DL}+1,0(\mathrm{~A}$ or $\mathrm{R})$ 
- $1,0 \mathrm{DL}+0,75 \mathrm{LL}+0,75(\mathrm{~A}$ or $\mathrm{R})$

- $1,0 \mathrm{DL} \pm(0,6 \mathrm{~W}$ or $0,7 \mathrm{E})$

- $1,0 \mathrm{DL} \pm 0,75(0,6 \mathrm{~W}$ or $0,75 \mathrm{E})+0,75 \mathrm{LL}+0,75(\mathrm{~A}$ or $\mathrm{R})$

- $0,6 \mathrm{DL} \pm 0,6 \mathrm{~W}$

- $0,6 \mathrm{DL} \pm 0,7 \mathrm{E}$

\subsection{Controlof Strengthand Stability}

- Deviation between levels

- $\quad$ Effect of P - $\Delta$

- Number offoundations

\section{Result and Discussion}

\subsection{Determine the Seismic Design Category (KDS)}

Building Location

Soil condition

Building Function

Risk Category

Priority Factor

Application of Indonesia's 2021 Design Response Spectrum:
: Mojokerto City, East Java Province, Indonesia

: Soft

: Hospital

: IV (table 3, SNI 1726-2019)

: 1,5 (table 4, SNI 1726-2019)

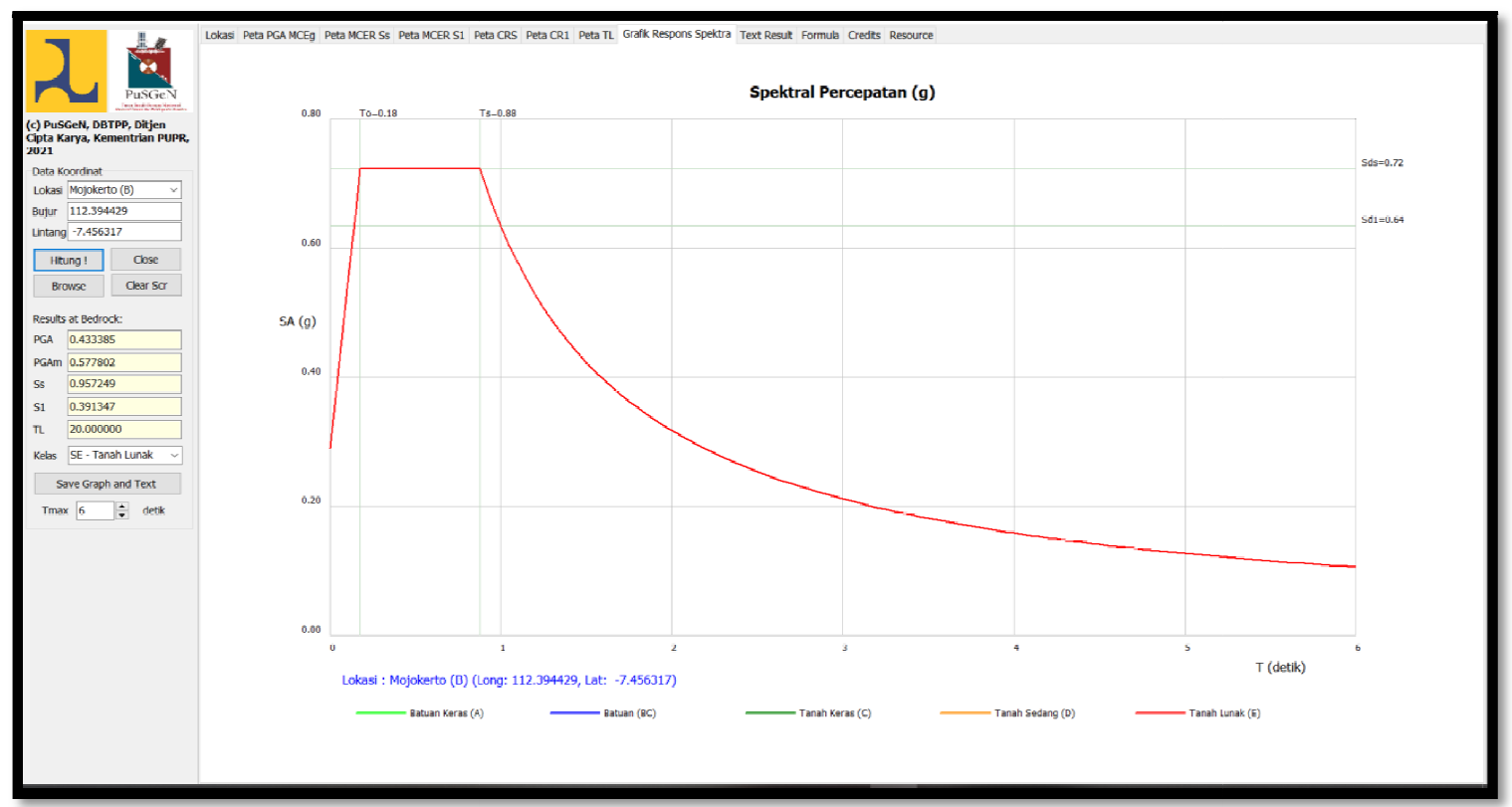

\section{Figure 1}

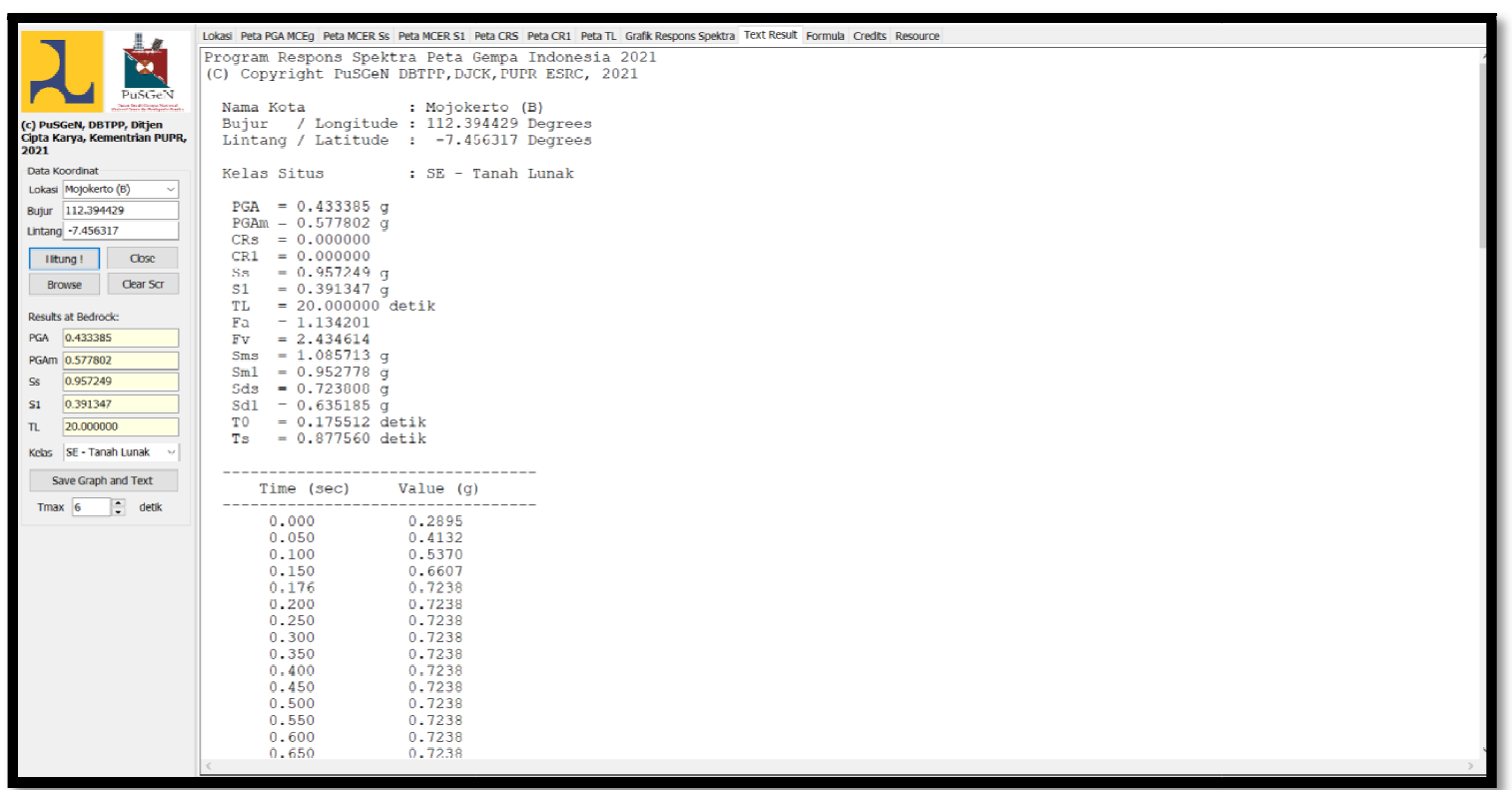

Figure 2 
Mojokerto Location : $\quad \mathrm{S}_{\mathrm{s}}=0,957 \mathrm{~g}$

$$
\begin{aligned}
& \mathrm{S}_{1}=0,391 \mathrm{~g} \\
& \mathrm{~F}_{\mathrm{a}}=1,134 \\
& \mathrm{~F}_{\mathrm{V}}=2,434 \\
& \mathrm{~S}_{\mathrm{DS}}=0,723 \mathrm{~g} \\
& \mathrm{~S}_{\mathrm{D} 1}=0,635 \mathrm{~g}
\end{aligned}
$$

\subsection{Determine the Natural Vibration TimeT}

For Mojokerto $\mathrm{S}_{\mathrm{D} 1}=0,635 \rightarrow \mathrm{C}_{\mathrm{U}}=1,4$

$\mathrm{T}_{\mathrm{a}}=\mathrm{C}_{\mathrm{t}} \mathrm{h}_{\mathrm{n}}$

$\mathrm{T}_{\mathrm{a}}=0,0466 \times 17^{0,9}=0,597 \mathrm{sec}$,

$\mathrm{T}_{\mathrm{a}}$ max allowable $=\mathrm{C}_{\mathrm{U}} \mathrm{T}_{\mathrm{a}}=1,4 \times 0,597=0,836 \mathrm{sec}$

Crack Condition

$\mathrm{T}_{\mathrm{c}}$ mode $1=0,952$

$\mathrm{T}_{\mathrm{c}}$ mode $2=0,906$

Un Crack Condition

$\mathrm{T}_{\mathrm{c}}$ mode $1=0,690$

$\mathrm{T}_{\mathrm{c}}$ mode $2=0,665$

Tmax $=0,836$

Used $\mathrm{T}_{\mathrm{x}}=0,836 ; \mathrm{T}_{\mathrm{y}}=0,836$

\subsection{Analysis of Equivalent Static}

The Basic Seismik Load $\rightarrow \mathrm{V}=\mathrm{C}_{\mathrm{S}} \mathrm{W}$

$\mathrm{S}_{\mathrm{D} 1}=0,635$

$\mathrm{S}_{\mathrm{Ds}}=0,723$

$\mathrm{T}_{\mathrm{X}}=0,836 \mathrm{sec}$,

$\mathrm{T}_{\mathrm{Y}}=0,836 \mathrm{sec}$

$\mathrm{R}_{\mathrm{X}}=8$

$\mathrm{R}_{\mathrm{Y}}=8$

$\mathrm{I}_{\mathrm{e}}=1,5$

\begin{tabular}{|c|c|c|c|c|}
\hline & $\mathbf{C}_{\mathbf{s} \text { min }}$ & $\mathbf{C}_{\mathbf{S}}$ & $\mathbf{C}_{\mathbf{S} \text { max }}$ & $\mathbf{C}_{\mathbf{S}}$ \\
\cline { 2 - 5 } & $0,044 \mathrm{~S}_{\mathrm{DS}} \mathrm{I}_{\mathrm{E}}$ & $\mathrm{S}_{\mathrm{DS}} /\left(\mathrm{R} / \mathrm{I}_{\mathrm{E}}\right)$ & $\mathrm{S}_{\mathrm{DS}} /\left[\mathrm{T}\left(\mathrm{R} / \mathrm{I}_{\mathrm{E}}\right)\right]$ & Value Selected \\
\hline X-direction & 0,048 & 0,135 & 0,162 & $\mathbf{0 , 1 3 5}$ \\
\hline Y-direction & 0,048 & 0,135 & 0,162 & $\mathbf{0 , 1 3 5}$ \\
\hline
\end{tabular}

Table 1

Distribution ofEquivalent Static Earthquake Force:

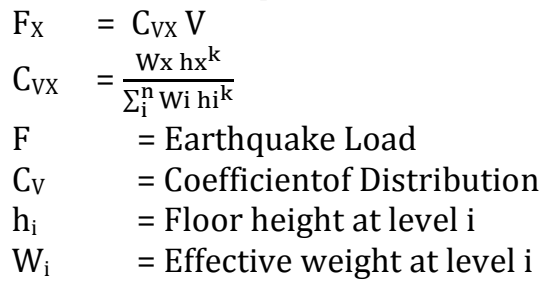

$\mathrm{K}$ for the equivalent static calculation:

\begin{tabular}{|c|c|}
\hline T (sec) & K \\
\hline $\mathrm{T} \leq 0,5$ & 1 \\
\hline $0,5<\mathrm{T}<2,5$ & Interpolation \\
\hline $\mathrm{T} \geq 2,5$ & 2 \\
\hline
\end{tabular}

$\mathrm{T}_{\mathrm{X}}=0,836 \rightarrow \mathrm{k}=1,168$

$\mathrm{T}_{\mathrm{Y}}=0,836 \rightarrow \mathrm{k}=1,168$

Earthquake Scale Factor $=(\mathrm{g} \times \mathrm{I}) / \mathrm{R}$ :

$\mathrm{R}_{\mathrm{x}}=8 \quad \mathrm{I}_{\mathrm{e}}=1,5$

$\mathrm{R}_{\mathrm{x}}=8 \quad \mathrm{I}_{\mathrm{e}}=1,5$

$\mathrm{g}($ gravitation $)=9,81 \mathrm{~m} / \mathrm{sec}^{2}$

\subsection{Analysisof Design Spectra Response}

Using Spectra Responsefrom Puskim in 2021

Earthquake ScaleFactor $=(\mathrm{g}$ x I $) / \mathrm{R}$ : 
$\mathrm{R}_{\mathrm{X}}=8 \quad \mathrm{I}_{\mathrm{e}}=1,5$

$\mathrm{R}_{\mathrm{Y}}=8 \quad \mathrm{I}_{\mathrm{e}}=1,5$

$\mathrm{g}$ (gravitation) $=9,81 \mathrm{~m} / \mathrm{sec}^{2}$

\subsection{Deflectionat Level X}

$\delta_{\mathrm{X}}=\frac{\mathrm{Cd} \delta \mathrm{xe}}{\mathrm{Ie}}$

$\mathrm{C}_{\mathrm{d}}=$ deflection enlargement factor, $\mathrm{x}$ direction $=5,5, \mathrm{y}$ direction $=5,5$

$\delta_{\mathrm{Xe}}=$ deflection determined byelasticanalysis

$\mathrm{I}_{\mathrm{e}}=$ the virtue factor $(1,5)$

Deviation :

\begin{tabular}{|c|c|c|c|c|c|c|c|c|}
\hline \multirow{3}{*}{ Floor } & \multirow{3}{*}{$\mathrm{h}(\mathrm{m})$} & \multicolumn{3}{|c|}{ Lateral Load, $\mathrm{X}$ direction } & \multicolumn{3}{|c|}{ Lateral Load, Y direction } & \multirow[b]{2}{*}{$\Delta \mathrm{a} / \rho$} \\
\hline & & $\delta_{X e}$ & $\delta x$ & $\Delta \mathrm{x}$ & $\delta y_{e}$ & $\delta y$ & $\Delta y$ & \\
\hline & & $(\mathrm{mm})$ & $(\mathrm{mm})$ & $(\mathrm{mm})$ & $(\mathrm{mm})$ & $(\mathrm{mm})$ & $(\mathrm{mm})$ & $(\mathrm{mm})$ \\
\hline Roof & 17 & 34,50 & 126,50 & 17,60 & 28,07 & 102,92 & 13,82 & 30,77 \\
\hline 4 & 13 & 29,70 & 108,90 & 29,33 & 24,30 & 89,10 & 23,36 & 30,77 \\
\hline 3 & 9 & 21,70 & 79,57 & 38,10 & 17,93 & 65,74 & 30,69 & 30,77 \\
\hline 2 & 5 & 11,31 & 41,47 & 41,47 & 9,56 & 35,05 & 35,05 & 38,46 \\
\hline
\end{tabular}

Table 3

\subsection{Determination of $P-\Delta$ Effect}

Coefficient ofstability ( $\theta$ ) can be calculated based on the following equation:

$$
\theta=\frac{\mathrm{Px} \Delta \mathrm{Ie}}{\mathrm{Vxhsx} C \mathrm{Cd}} \theta_{\text {mak }}=0,5 /\left(1 \times \mathrm{C}_{\mathrm{d}}\right)
$$

Calculation of $\theta$ due to the influence of $\mathrm{P}-\Delta$

$\mathrm{X}$ direction

\begin{tabular}{|c|c|c|c|c|c|c|}
\hline Floor & $\mathrm{h}(\mathrm{m})$ & $\mathrm{P}(\mathrm{kN})$ & $\Delta \mathrm{x}(\mathrm{mm})$ & $\mathrm{Vx}(\mathrm{kN})$ & $\Theta$ & $\theta$ max \\
\hline 4th floor & 4 & 4283 & 17,60 & 883 & 0,0039 & 0,091 \\
\hline 3rd floor & 4 & 9969 & 29,33 & 1574 & 0,0084 & 0,091 \\
\hline 2nd floor & 4 & 15656 & 38,10 & 2079 & 0,0130 & 0,091 \\
\hline 1st floor & 5 & 21473 & 41,47 & 2367 & 0,0137 & 0,091 \\
\hline
\end{tabular}

Table 4

Y direction

\begin{tabular}{|c|c|c|c|c|c|c|}
\hline Floor & $\mathrm{h}(\mathrm{m})$ & $\mathrm{P}(\mathrm{kN})$ & $\Delta \mathrm{y}(\mathrm{mm})$ & $\mathrm{Vy}(\mathrm{kN})$ & $\Theta$ & $\theta \max$ \\
\hline 4th floor & 4 & 4283 & 13,82 & 886 & 0,0030 & 0,091 \\
\hline 3rd floor & 4 & 9969 & 23,36 & 1588 & 0,0067 & 0,091 \\
\hline 2nd floor & 4 & 15656 & 30,69 & 2104 & 0,0104 & 0,091 \\
\hline 1st floor & 5 & 21473 & 35,05 & 2404 & 0,0114 & 0,091 \\
\hline
\end{tabular}

Table 5

$\Theta<\Theta_{\text {mak, }}$ then the effect of $\mathrm{P}-\Delta$ was ignored

\subsection{Foundation Workload}

\subsubsection{Due to Load 1DL+1LL}

\begin{tabular}{|c|c|c|c|c|c|c|c|c|c|c|c|c|c|c|c|}
\hline $\begin{array}{l}\text { Joint } \\
\text { Text }\end{array}$ & $\begin{array}{c}\text { OutputCase } \\
\text { Text }\end{array}$ & $\begin{array}{l}\mathbf{P} \\
\text { ton }\end{array}$ & $\begin{array}{l}M \mathbf{M} \\
\text { ton-m }\end{array}$ & $\begin{array}{l}\text { My } \\
\text { ton-m }\end{array}$ & $\begin{array}{l}\text { Jumlah } \\
\text { Pile (n) }\end{array}$ & $X \max$ & Ymax & $\sum X^{2}$ & $\sum Y^{2}$ & $P / n$ & $\frac{(\text { Mx.Xmax) }}{\sum X^{2}}$ & $\frac{1 y \cdot Y \max )}{\sum Y^{2}}$ & $\begin{array}{l}\text { P terjadi } \\
\text { (ton/pile) }\end{array}$ & $\begin{array}{c}P \text { l lin } \\
65 \text { ton } x \text { Eff }\end{array}$ & Eff \\
\hline 126 & $1 \mathrm{DL}+1 \mathrm{LL}$ & 125,12 & 2,27 & 5,63 & 4,00 & 0,50 & 0,50 & 0,75 & 0,50 & 31,28 & 1,51 & 5,63 & 38,42 & 51,35 & 0,790 \\
\hline 128 & $1 \mathrm{DL}+1 \mathrm{LL}$ & 143,38 & 08 & 86 & 00 &, 75 & 0,75 & 2,25 & 2,25 &, 68 & 0,69 & 1,95 & 31,32 & 45,11 & 0,694 \\
\hline 130 & $1 \mathrm{DL}+1 \mathrm{LL}$ & 126,14 &, 21 & 4,41 & 4,00 & 0,50 & 0,50 & 0,75 & 0,50 & 1,53 & 2,80 & 4,41 & 38,75 & 50,70 & 0,780 \\
\hline 132 & $1 \mathrm{DL}+1 \mathrm{LL}$ & 261,37 & 1,05 & 0,94 & 7,00 & 0,75 & 0,75 & 2,25 & 2,25 & 37,34 & 0,35 & 0,31 & 38,00 & 45,11 & 0,694 \\
\hline 134 & $1 \mathrm{DL}+1 \mathrm{LL}$ & 220,83 & 3,96 & 4,12 & 7,00 & 0,75 & 0,75 & 2,25 & 2,25 & 31,55 & 1,32 & 1,37 & 34,24 & 45,11 & 0,694 \\
\hline 136 & $1 \mathrm{DL}+1 \mathrm{LL}$ & 185,33 & 0,17 & 2,73 & 5,00 & 0,75 & 0,75 & 2,25 & 2,25 & 37,07 & 0,06 & 0,91 & 38,03 & 45,11 & 0,694 \\
\hline 138 & $1 \mathrm{DL}+1 \mathrm{LL}$ & 318,43 & 0,11 & 1,24 & 8,00 & 0,75 & 0,75 & 3,38 & 2,25 & 39,80 & 0,03 & 0,41 & 40,24 & 44,01 & 0,677 \\
\hline 139 & $1 \mathrm{DL}+1 \mathrm{LL}$ & 205,45 & 0,58 & 2,19 & 7,00 & 0,75 & 0,75 & 2,25 & 2,25 & 29,35 & 0,19 & 0,73 & 30,27 & 45,11 & 0,694 \\
\hline 141 & $1 \mathrm{DL}+1 \mathrm{LL}$ & 117,17 & 4,41 & 4,07 & 4,00 & 0,50 & 0,50 & 0,75 & 0,50 & 29,29 & 2,9 & 4,07 & 36,31 & 50,70 & 0,780 \\
\hline 143 & $1 \mathrm{DL}+1 \mathrm{LL}$ & 210,32 & 2,05 & 0,45 & 6,00 & 0,75 & 0,75 & 2,25 & 2,25 & 35,05 & 0,68 & 0,15 & 35,89 & 45,11 & 0,694 \\
\hline 145 & $1 \mathrm{DL}+1 \mathrm{LL}$ & 145,06 & 3,50 & 3,82 & 5,00 & 0,75 & 0,75 & 1,69 & 1,13 & 29,01 & 1,55 & 2,55 & 33,11 & 45,11 & 0,694 \\
\hline 147 & $1 \mathrm{DL}+1 \mathrm{LL}$ & 41,12 & 1,07 & 5,49 & 2,00 & 0,50 & & 0,50 & & 20,56 & 1,07 & 1,37 & 23,01 & 58,31 & 0,897 \\
\hline 149 & $1 \mathrm{DL}+1$ & 85,35 & 0,8 & 1,0 & 3,00 & 0,60 & 0,60 & 0,72 & 0,72 & 28,45 & 0,00 & 0,87 & 29,32 & 49,01 & 0,754 \\
\hline 200 & $1 \mathrm{DL}+1 \mathrm{LL}$ & 62,40 & 0,64 & 6,35 & 2,00 & 0,50 & & 0,50 & & 31,20 & & 1,59 & 32,79 & 58,31 & 0,897 \\
\hline
\end{tabular}

Table 6 


\subsubsection{Due to Load1DL+0,75LL+0,525RSPX+0,157RSPY}

\begin{tabular}{|c|c|c|c|c|c|c|c|c|c|c|c|c|c|c|c|}
\hline $\begin{array}{l}\text { Joint } \\
\text { Text }\end{array}$ & $\begin{array}{l}\text { OutputCase } \\
\text { Text }\end{array}$ & $\begin{array}{l}\mathbf{P} \\
\text { ton }\end{array}$ & $\begin{array}{l}\text { Mx } \\
\text { ton-m }\end{array}$ & $\begin{array}{l}\text { My } \\
\text { ton-m }\end{array}$ & $\begin{array}{l}\text { Jumlah } \\
\text { Pile (n) }\end{array}$ & $X \max$ & Ymax & $\sum X^{2}$ & $\sum Y^{2}$ & $\mathrm{P} / \mathrm{n}$ & $\frac{(M x \cdot X \max )}{\sum X^{2}}$ & $\frac{(M y \cdot Y \max )}{\sum Y^{2}}$ & $\begin{array}{l}\text { P terjadi } \\
\text { (ton/pile) }\end{array}$ & $\begin{array}{c}\text { P ljin } \\
65 \times 1,3 \text { ton } x \text { Eff }\end{array}$ & Eff \\
\hline 126 & $1 \mathrm{DL}+0,75 \mathrm{LL}+0,525 \mathrm{RSPX}+$ & 125,22 & 8,96 & 23,62 & 4,00 & 0,50 & 0,50 & 0,75 & 0,50 & 31,30 & 5,97 & 23,62 & 60,90 & 66,76 & 0,790 \\
\hline 128 & $1 \mathrm{DL}+0,75 \mathrm{LL}+0,525 \mathrm{RSPX}+$ & 158,00 & 11,65 & 33,67 & 5,00 & 0,75 & 0,75 & 2,25 & 2,25 & 31,60 & 3,88 & 11,22 & 46,71 & 58,64 & 0,694 \\
\hline 130 & $1 \mathrm{DL}+0,75 \mathrm{LL}+0,525 \mathrm{RSPX}+$ & 135,98 & 12,81 & 19,88 & 4,00 & 0,50 & 0,50 & 0,75 & 0,50 & 33,99 & 8,54 & 19,88 & 62,42 & 65,91 & 0,780 \\
\hline 132 & $1 \mathrm{DL}+0,75 L \mathrm{~L}+0,525 \mathrm{RSPX}+$ & 253,16 & 8,83 & 27,32 & 7,00 & 0,75 & 0,75 & 2,25 & 2,25 & 36,17 & 2,94 & 9,11 & 48,22 & 58,64 & 0,694 \\
\hline 134 & $1 D L+0,75 L L+0,525 R S P X+$ & 230,08 & 14,63 & 30,36 & 7,00 & 0,75 & 0,75 & 2,25 & 2,25 & 32,87 & 4,88 & 10,12 & 47,87 & 58,64 & 0,694 \\
\hline 136 & $1 D L+0,75 L L+0,525 R S P X+$ & 191,63 & 8,73 & 20,88 & 5,00 & 0,75 & 0,75 & 2,25 & 2,25 & 38,33 & 2,91 & 6,96 & 48,20 & 58,64 & 0,694 \\
\hline 138 & $1 \mathrm{DL}+0,75 \mathrm{LL}+0,525 \mathrm{RSPX}+$ & 309,27 & 8,10 & 26,23 & 8,00 & 0,75 & 0,75 & 3,38 & 2,25 & 38,66 & 1,80 & 8,74 & 49,20 & 57,21 & 0,677 \\
\hline 139 & $1 \mathrm{DL}+0,75 \mathrm{LL}+0,525 \mathrm{RSPX}+$ & 214,82 & 11,66 & 27,76 & 7,00 & 0,75 & 0,75 & 2,25 & 2,25 & 30,69 & 3,89 & 9,25 & 43,83 & 58,64 & 0,694 \\
\hline 141 & $1 \mathrm{DL}+0,75 \mathrm{LL}+0,525 \mathrm{RSPX}+$ & 127,45 & 3,90 & 22,55 & 4,00 & 0,50 & 0,50 & 0,75 & 0,50 & 31,86 & 2,60 & 22,55 & 57,01 & 65,91 & 0,780 \\
\hline 143 & $1 \mathrm{DL}+0,75 L \mathrm{~L}+0,525 \mathrm{RSPX}+$ & 209,62 & 5,41 & 30,35 & 6,00 & 0,75 & 0,75 & 2,25 & 2,25 & 34,94 & 1,80 & 10,12 & 46,86 & 58,64 & 0,694 \\
\hline 145 & $1 \mathrm{DL}+0,75 \mathrm{LL}+0,525 \mathrm{RSPX}+$ & 160,85 & 6,97 & 32,60 & 5,00 & 0,75 & 0,75 & 1,69 & 1,13 & 32,17 & 3,10 & 21,74 & 57,01 & 58,64 & 0,694 \\
\hline 147 & $1 \mathrm{DL}+0,75 \mathrm{LL}+0,525 \mathrm{RSPX}+$ & 53,93 & 2,87 & 8,85 & 2,00 & 0,50 & & 0,50 & & 26,97 & 2,87 & 2,21 & 32,05 & 75,80 & 0,897 \\
\hline 149 & $1 \mathrm{DL}+0,75 \mathrm{LL}+0,525 \mathrm{RSPX}+$ & 93,67 & 2,73 & 16,22 & 3,00 & 0,60 & 0,60 & 0,72 & 0,72 & 31,22 & 0,00 & 13,52 & 44,74 & 63,71 & 0,754 \\
\hline 200 & $1 \mathrm{DL}+0,75 L \mathrm{~L}+0,525 \mathrm{RSPX}+$ & 78,44 & 4,37 & 22,06 & 2,00 & 0,50 & & 0,50 & & 39,22 & & 5,51 & 44,73 & 75,80 & 0,897 \\
\hline
\end{tabular}

Table 7

\subsubsection{Due to Load1DL+0, 75LL+0,525RSPY+0,157RSPX}

\begin{tabular}{|c|c|c|c|c|c|c|c|c|c|c|c|c|c|c|c|}
\hline $\begin{array}{r}\text { Joint } \\
\text { Text }\end{array}$ & $\begin{array}{c}\text { OutputCase } \\
\text { Text }\end{array}$ & $\begin{array}{c}\mathbf{P} \\
\text { ton }\end{array}$ & $\underset{\text { ton-m }}{\mathrm{Mx}}$ & $\begin{array}{c}\text { My } \\
\text { ton-m }\end{array}$ & $\begin{array}{l}\text { Jumlah } \\
\text { Pile (n) }\end{array}$ & $X \max$ & Ymax & $\sum X^{2}$ & $\sum Y^{2}$ & $P / n$ & $\frac{(M x . X \max }{\sum X^{2}}$ & $\frac{(M y \cdot Y \max )}{\sum Y^{2}}$ & $\begin{array}{l}\text { P terjadi } \\
\text { (ton/pile) }\end{array}$ & $\begin{array}{c}\text { P ljin } \\
65 \times 1,3 \text { ton } \times E f f\end{array}$ & Eff \\
\hline 126 & $1 S W+1 D L+0,75 L L+0,5$ & 132,17 & 23,93 & 11,08 & 4,00 & 0,50 & 0,50 & 0,75 & 0,50 & 33,04 & 15,96 & 11,08 & 60,08 & 66,76 & 0,790 \\
\hline 128 & $1 S W+1 D L+0,75 L L+0,5$ & 154,11 & 28,63 & 21,89 & 5,00 & 0,75 & 0,75 & 2,25 & 2,25 & 30,82 & 9,54 & 7,30 & 47,66 & 58,64 & 0,694 \\
\hline 130 & $1 S W+1 D L+0,75 L L+0,5$ & 129,72 & 27,14 & 6,22 & 4,00 & 0,50 & 0,50 & 0,75 & 0,50 & 32,43 & 18,09 & 6,22 & 56,74 & 65,91 & 0,780 \\
\hline 132 & $1 S W+1 D L+0,75 L L+0,5$ & 252,23 & 26,12 & 11,63 & 7,00 & 0,75 & 0,75 & 2,25 & 2,25 & 36,03 & 8,71 & 3,88 & 48,62 & 58,64 & 0,694 \\
\hline 134 & $1 S W+1 D L+0,75 L L+0,5$ & 220,10 & 34,11 & 15,75 & 7,00 & 0,75 & 0,75 & 2,25 & 2,25 & 31,44 & 11,37 & 5,25 & 48,06 & 58,64 & 0,694 \\
\hline 136 & $1 S W+1 D L+0,75 L L+0,5$ & 183,44 & 23,85 & 5,37 & 5,00 & 0,75 & 0,75 & 2,25 & 2,25 & 36,69 & 7,95 & 1,79 & 46,43 & 58,64 & 0,694 \\
\hline 138 & $1 S W+1 D L+0,75 L L+0,5$ & 306,89 & 26,30 & 8,30 & 8,00 & 0,75 & 0,75 & 3,38 & 2,25 & 38,36 & 5,84 & 2,77 & 46,97 & 57,21 & 0,677 \\
\hline 139 & $1 S W+1 D L+0,75 L L+0,5$ & 204,40 & 31,87 & 11,31 & 7,00 & 0,75 & 0,75 & 2,25 & 2,25 & 29,20 & 10,62 & 3,77 & 43,59 & 58,64 & 0,694 \\
\hline 141 & $1 S W+1 D L+0,75 L L+0,5$ & 122,10 & 18,43 & 9,74 & 4,00 & 0,50 & 0,50 & 0,75 & 0,50 & 30,52 & 12,28 & 9,74 & 52,55 & 65,91 & 0,780 \\
\hline 143 & $1 S W+1 D L+0,75 L L+0,5$ & 210,95 & 22,87 & 15,34 & 6,00 & 0,75 & 0,75 & 2,25 & 2,25 & 35,16 & 7,62 & 5,11 & 47,90 & 58,64 & 0,694 \\
\hline 145 & $1 S W+1 D L+0,75 L L+0,5$ & 157,70 & 26,47 & 18,98 & 5,00 & 0,75 & 0,75 & 1,69 & 1,13 & 31,54 & 11,76 & 12,65 & 55,96 & 58,64 & 0,694 \\
\hline 147 & $1 S W+1 D L+0,75 L L+0,5$ & 60,67 & 9,54 & 2,72 & 2,00 & 0,50 & & 0,50 & & 30,34 & 9,54 & 0,68 & 40,56 & 75,80 & 0,897 \\
\hline 149 & $1 S W+1 D L+0,75 L L+0,5$ & 106,11 & 10,91 & 8,95 & 3,00 & 0,60 & 0,60 & 0,72 & 0,72 & 35,37 & 0,00 & 7,46 & 42,83 & 63,71 & 0,754 \\
\hline 200 & $1 S W+1 D L+0,75 L L+0,5$ & 85,13 & 13,53 & 15,23 & 2,00 & 0,50 & & 0,50 & & 42,56 & & 3,81 & 46,37 & 75,80 & 0,897 \\
\hline
\end{tabular}

Table 8

\section{Conclusion}

From the results of the evaluation, the following conclusions can be drawn

\subsection{The Deviation between Levels}

\begin{tabular}{|c|c|c|c|}
\hline Floor & $\boldsymbol{\Delta x}(\mathbf{m m})$ & $\boldsymbol{\Delta y}(\mathbf{m m})$ & $\boldsymbol{\Delta a} / \boldsymbol{\rho}(\mathbf{m m})$ \\
\hline $4^{\text {th }}$ & 17,60 & 13,82 & 30,77 \\
\hline $3^{\text {rd }}$ & 29,33 & 23,26 & 30,77 \\
\hline $2^{\text {nd }}$ & 38,10 & 30,69 & 30,77 \\
\hline $1^{\text {st }}$ & 41,47 & 35,05 & 38,46 \\
\hline
\end{tabular}

Table 9

4.2. The Effect ofP - $\Delta$ between Levels

\begin{tabular}{|c|c|c|c|}
\hline Floor & $\boldsymbol{\theta x}$ & $\boldsymbol{\theta y}$ & $\boldsymbol{\theta m a x}$ \\
\hline $4^{\text {th }}$ & 0,0039 & 0,0030 & 0,091 \\
\hline $3^{\text {rd }}$ & 0,0084 & 0,0067 & 0,091 \\
\hline $2^{\text {nd }}$ & 0,0130 & 0,0104 & 0,091 \\
\hline $1^{\text {st }}$ & 0,0137 & 0,0114 & 0,091 \\
\hline
\end{tabular}

Table 10 


\subsection{Foundation Workload}

\subsubsection{Due to Load 1DL+1LL}

\begin{tabular}{|c|c|c|}
\hline Joint & Workload (Ton) & Allowable Carrying Capacity (Ton) \\
\hline 126 & 38,42 & 51,35 \\
\hline 128 & 31,32 & 45,11 \\
\hline 130 & 38,75 & 50,70 \\
\hline 132 & 38,00 & 45,11 \\
\hline 134 & 34,24 & 45,11 \\
\hline 136 & 38,03 & 45,11 \\
\hline 138 & 40,24 & 44,01 \\
\hline 139 & 30,27 & 45,11 \\
\hline 141 & 36,31 & 50,70 \\
\hline 143 & 35,89 & 45,11 \\
\hline 145 & 33,11 & 45,11 \\
\hline 147 & 23,01 & 58,31 \\
\hline 149 & 29,32 & 49,01 \\
\hline 200 & 32,79 & 58,31 \\
\hline
\end{tabular}

Table 11

4.3.2. Due to Load 1DL+0, 75LL+0,525RSPX+0,157RSPY

\begin{tabular}{|c|c|c|}
\hline Joint & Workload (Ton) & Allowable Carrying Capacity (Ton) \\
\hline 126 & 60,90 & 66,76 \\
\hline 128 & 46,71 & 58,64 \\
\hline 130 & 62,42 & 65,91 \\
\hline 132 & 48,22 & 58,64 \\
\hline 134 & 47,87 & 58,64 \\
\hline 136 & 48,20 & 58,64 \\
\hline 138 & 49,20 & 57,21 \\
\hline 139 & 43,83 & 58,64 \\
\hline 141 & 57,01 & 65,91 \\
\hline 143 & 46,86 & 58,64 \\
\hline 145 & 57,01 & 58,64 \\
\hline 147 & 32,05 & 76,80 \\
\hline 149 & 44,47 & 63,71 \\
\hline 200 & 44,73 & 75,80 \\
\hline
\end{tabular}

Table 12

4.3.3. Due to load 1DL+0,75LL+0,525RSPY+0,157RSPX

\begin{tabular}{|c|c|c|}
\hline Joint & Workload (ton) & Allowable Carrying Capacity (ton) \\
\hline 126 & 60,08 & 66,76 \\
\hline 128 & 47,66 & 58,64 \\
\hline 130 & 56,74 & 65,91 \\
\hline 132 & 48,62 & 58,64 \\
\hline 134 & 48,06 & 58,64 \\
\hline 136 & 46,43 & 58,64 \\
\hline 138 & 46,97 & 57,21 \\
\hline 139 & 43,59 & 58,64 \\
\hline 141 & 52,55 & 65,91 \\
\hline 143 & 47,90 & 58,64 \\
\hline 145 & 55,96 & 58,64 \\
\hline 147 & 40,56 & 75,80 \\
\hline 149 & 42,83 & 63,71 \\
\hline 200 & 46,37 & 75,80 \\
\hline
\end{tabular}


4.3.4. From the Calculation Results It Can Be Concluded as Follows

- The deviation between levels in the X direction on the 1st floor and the 2nd floor is greater than the allowable deviation, while on the 3rd and 4th floors it is smaller than allowed. The deviation between the levels in the $\mathrm{Y}$ direction of all floors is less than the allowable.

- The effect of P - $\Delta$ on all floors is less than the allowable.

- Work load on the foundation at all points of the column with a combination of gravity load and earthquake load is less than the allowable bearing capacity.

\section{References}

i. Afnan, Yucha Al Kautsar; M. AfifShulhan, M. Afif; and Iskandar Yasin, Iskandar (2020). Perbandingan Respons Spektrum Gempa Antara SNI 1726-2012 Dan SNI 1726-2019 Di Indonesia. JurnalUniversitas SarjanawiyataTamansiswa. jurnal.ustjogja.ac.id.

ii. Istiono, Heri; Khoe, Letisia (2020). Analisis Perhitungan Struktur Bangunan Tahan Gempadengan KolamRenang Berdasarkan SNI 1726:2019. JurnalTeknikSipil, Vol 1, No 2, November 2020. ISSN (Online) 2747-0733. $146-151$.

iii. Siddiq, Suwandojo (2014). BangunanTahan Gempa Berbasis Standar Nasional Indonesia. Jurnal Standardisasi. ISSN: 1411-0822. E-ISSN: 2337-5833. https://js.bsn.go.id/index.php/standardisasi/article/view/664/351

iv. Tata caraperencanaanketahanangempauntukstrukturbangunanged ungdan non gedungSNI 1726-2012.

v. Tata caraperencanaanketahanangempauntukstrukturbangunangedungdan non gedungSNI 1726-2019.

vi. AplikasiSpektrumResponsDesain Indonesia 2021.

vii. Dokumen Perencanaan Gedung Rumah Sakit Basuni Mojokerto

viii. Soil Test 